\title{
Sobre o planejamento e a (DES)articulação das políticas públicas no Brasil: a saúde e o meio ambiente
}

\section{(DIS)articulation of planning and public policies in Brazil: the health and environmental sectors}

DOI: $10.46919 / \operatorname{archv} 1 n 6-019$

Recebimento dos originais: 01/09/2020

Aceitação para publicação: 30/10/2020

\section{Mariana Matera Veras}

Formação acadêmica mais alta: Doutor em Fisiopatologia

Instituição: Universidade de São Paulo

Endereço: LPAE- LIM05 FMUSP Av Dr Arnaldo 455 sala 1304, Cerqueira Cesar São Paulo SP CEP 01246903

E-mail: verasine@usp.br

\section{José Guilherme V. Closs}

Formação acadêmica mais alta: Graduando em história Instituição: Universidade de São Paulo

Endereço: LPAE- LIM05 FMUSP Av Dr Arnaldo 455 sala 1304, Cerqueira Cesar São Paulo SP CEP 01246903

E-mail: joseveras@usp.br

\section{Vcitor Yuri Yariwake}

Formação acadêmica mais alta: Mestre em Fisiopatologia

Instituição: Universidade de São Paulo

Endereço: LPAE- LIM05 FMUSP Av Dr Arnaldo 455 sala 1304, Cerqueira Cesar São Paulo SP CEP 01246903

E-mail: victoriyuji.13@gmail.com

\section{Paulo H. N. Saldiva}

Formação acadêmica mais alta: Professor titular em Patologia

Instituição: Universidade de São Paulo

Endereço: LPAE- LIM05 FMUSP Av Dr Arnaldo 455 sala 1304, Cerqueira Cesar São Paulo SP CEP 01246903

E-mail:pepino@usp.br

\section{Nathalia Villa dos Santos}

Formação acadêmica mais alta: Doutor em Patologia Instituição: Universidade de São Paulo

Endereço: LPAE- LIM05 FMUSP Av Dr Arnaldo 455 sala 1304, Cerqueira Cesar São Paulo SP CEP 01246903

E-mail: nathvilla@gmail.com 


\title{
Lais Fajerstzjan
}

Formação acadêmica mais alta: Doutor em Patologia

Instituição: Universidade de São Paulo

\author{
Endereço: LPAE- LIM05 FMUSP Av Dr Arnaldo 455 sala 1304, Cerqueira Cesar São Paulo SP CEP \\ 01246903 \\ E-mail: laisfajer@gmail.com
}

\begin{abstract}
RESUMO
Em 2001, a Organização Mundial da Saúde já indicava que a saúde deveria ser prioridade nas políticas das nações já que é o principal fator estrutural para o crescimento econômico e para a redução da pobreza. Muitos fatores interagem de forma a determinar ou influenciar o estado de saúde de um indivíduo ou comunidade havendo, portanto, diversas oportunidades de intervenção positiva das diferentes políticas públicas sobre a saúde sejam elas de meio ambiente, transporte ou econômicas. Prever todos os impactos das diferentes políticas sobre a saúde é muito difícil, entretanto, a inclusão de aspectos relacionados à saúde na elaboração e no planejamento de novas políticas contribuiria de forma efetiva para a melhoria e promoção da saúde em todos os níveis sociais. Neste estudo analisamos com base na literatura científica, documentos oficiais do governo e na mídia impressa duas situações onde a falta de comunicação, aproximação e engajamento dos diferentes setores e pastas podem comprometer diretamente a saúde da população brasileira, além de prejudicar resultados de planos e políticas anteriores. As duas situações selecionadas para este estudo referem - se ao momento da tomada de decisão ou, ao da formulação de uma política pública de saúde específica. A responsabilidade sobre a vida humana deve estar à frente dos interesses econômicos e isto exige que o meio ambiente como determinante da saúde e a saúde por si sejam levados em consideração no s planos de ação estatal, seja no curto, médio e longo prazo. As autoridades governamentais e os grandes empresários devem assumir esse ônus (econômico) em conjunto com a sociedade, redirecionando a política econômica de suas decisões ao bem da vida e à saúde da população. A situação de desconsideração das questões de saúde e meio ambiente podem ser parcialmente explicadas pelo modelo político-econômico brasileiro que favorece processos produtivos mesmo que estes impliquem em danos diretos ou indiretos sobre a saúde e pela falta de preparo técnico de gestores e profissionais da saúde na avaliação dos impactos sobre a saúde das políticas e ações de outros setores e para assumir a necessidade de ações inter setoriais. As ações do governo e suas consequências sobre o bem-estar e saúde da população requerem hoje um novo modelo de governança no qual um olhar mais holístico é necessário sendo o "método da Saúde em todas as Políticas" uma forma já testada em outros países, para orientar e auxiliar líderes e formuladores de políticas a integrar a o aspecto saúde durante o desenvolvimento, implementação e avaliação das políticas públicas e serviços prestados.
\end{abstract}

Palavras Chaves: saúde, meio ambiente, políticas públicas.

\begin{abstract}
In 2001, The World Health Organization has highlighted that health should be the priority in National polices, because health is the principal structural factor for economic development and poverty reduction. Different factors interact to determine and affect the health status of individuals and communities, in a way that there are many opportunities for positive intervention of different public policies of environment, transport and energy generation on health. Forecast of all the health impacts from different policies is almost impossible; however, the inclusion of the health aspects during the process of policies formulation and planning would effectively contribute for health promotion in all social levels. In this study, we have analyzed based on scientific articles, governmental official documents and press media (journals from two cases where the absence of communication, poor engagement between different government sectors could affect the health of the Brazilian population and compromised results of previous policies and plans. The two cases selected for this study refer to a public policy formulation and
\end{abstract}


a decision making The two situations selected for this study refer to the time of decision making (economic sector) and to the formulation of a specific public policy for health. The responsibility for the human health should be ahead of economic interests and this requires that the environment as a health determinant and health per se to be considered in all State action plan, both in the short, medium and long term. Governmental authorities and big companies should assume the (economic) burden together with the society to redirect the economic policy decisions to protect and promote health. In Brazil, the diminished importance of health issues and environmental protection during the decision making process can be partly explained by: 1) the Brazilian political economic model that favors the productive processes even if it implicates in direct or indirect impacts on health; 2) the lack of technical preparation for the assessment of health impacts of policies and actions 3) rare recognition by the federal, state and municipal governments that all policies need sectoral planning. The interdependence of government actions and their consequences on the wellbeing of the population require a new model of governance with a more holistic look. The Health in All Policies method would be an option already tested in other countries to guide and assist leaders and policy makers to integrate the health aspect in the development, implementation and evaluation of public policies and services.

Keywords: health, environment, public police

\section{INTRODUÇÃO}

Profissionais da saúde e formuladores de políticas públicas não alcançarão progressos significativos para os desafios atuais de saúde pública sem o envolvimento dos diversos setores do governo e seus parceiros tais como educadores, planejadores, empregadores e empresários (McGinnis et al., 2002).

O setor da saúde, seja municipal, estadual ou federal, enfrenta hoje complexos desafios em sua gestão, na promoção da saúde e melhoria na qualidade de vida devido a tomadas de decisão de outros setores aparentemente não relacionados ao setor da saúde. As políticas públicas, ou sociais, econômicas, agrícolas, de uso e ocupação de solo, transporte, meio ambiente entre tantas outras, incluindo a de qualidade na educação moldam e afetam direta e indiretamente os desfechos em saúde e atingem toda a população.

Há um crescente reconhecimento da importância da saúde para as estratégias de crescimento e desenvolvimento econômico, para a competitividade e para o desenvolvimento sustentável. Em 2001, a Organização Mundial da Saúde (OMS) já indicava que a saúde deveria ser uma prioridade nas políticas das nações já que é o principal fator e estrutural para o crescimento econômico e para a redução da pobreza (WHO, 2001). Entretanto a importância do investimento direto e indireto em saúde (por exemplo, relacionado à preservação de áreas verdes e florestas endêmicas) é subestimada por governantes e por grandes empresários principalmente em países subdesenvolvidos ou em desenvolvimento (Wilkinson et al., 2006). 
Outro destaque é que muitos fatores interagem de forma a determinar ou influenciar o estado de saúde de um indivíduo ou comunidade; de forma ampla os fatores mais impactantes dizem respeito ao local onde vivemos, a qualidade do meio ambiente, a renda, o nível de educação e a genética; ao contrário do que a maioria das pessoas acreditam ser o acesso a serviços de saúde o determinante da saúde para uma população (WHO, 2015).

Diversas são as oportunidades de intervenção positiva das diferentes políticas públicas sobre a saúde, sejam elas de meio ambiente, transporte ou econômicas. Por exemplo, políticas de redução na emissão de gases estufa podem ter co-beneficios sobre a saúde na medida em que promovem a redução de poluentes atmosféricos em nível local, sabidamente capazes de provocar efeitos de curto e longo prazo sobre a saúde (Haines et al., 2009, Woodckook et al., 2009; Marandaya et al., 2009; Wilkinson et al., 2009; Friel et al., 2009). Em pelo menos quatro setores (energia elétrica, transporte, agricultura e combustíveis) há potencial para se agregar políticas de saúde e ambientais em um mesmo objetivo (Haines et al., 2009). Medidas que estimulem modalidades de transporte ativo, como caminhar e andar de bicicleta, não apenas reduzem a emissão de gases estufa (por veículos automotores) como também melhoram a saúde cárdio-respiratória (Woodckook et al., 2009).

O tema saúde é muito complexo e é bem difícil prever todos os impactos das diferentes políticas sobre a saúde. Entretanto, a inclusão de aspectos relacionados a saúde na elaboração e no planejamento de novas políticas contribuiria de forma efetiva para a melhoria e promoção da saúde em todos os níveis sociais.

Pesquisas mostram que diferenças no cuidado da saúde são responsáveis por menos de $10 \%$ na variabilidade para morte prematura enquanto que fatores sociais, comportamentais e ambientais são responsáveis por 60\% desta variabilidade (McGinnis et al., 2002). Além disto, é importante salientar que a população está envelhecendo, e o impacto disso no desenvolvimento social e econômico vai depender da habilidade dos Estados em manter seus cidadãos saudáveis e ativos, diminuindo o impacto ao sistema de aposentadorias e seguro social. Em 40 anos (2010-2050) é esperado um crescimento de $56 \%$ no número de pessoas com mais de 60 anos nas regiões mais desenvolvidas e de $224 \%$ nas regiões menos desenvolvidas, o que representaria um crescimento também nas doenças crônicas não transmissíveis (WHO, 2005) Embora a ocorrência de doenças crônicas não seja restrita a faixa mais idosa da população, um maior número de idosos pode resultar em uma maior incidência de doenças crônicas não transmissíveis, tais como as cardiovasculares (ex: hipertensão), obesidade, diabetes, doenças neurodegenerativas que por sua vez representam uma sobrecarga na economia (WHO, 2005; Strong et al, 2005). 
Nos encontros realizados entre nações em Adelaide na Austrália, em 1988 e 2010 pela (WHO, 1988, 2010), reforça-se a recomendação de garantir que a saúde seja levada em consideração na formação e implementação de políticas, programas, projetos e planos de todos os setores. A meta é integrar questões de saúde nos processos de tomada de decisão em diferentes setores e pastas, não a tornando o ponto central, mas sim destacando sua importância e a necessidade de colaboração entre setores para que objetivos comuns sejam alcançados. Formas de se criar uma nação mais saudável, e ensinar as lideranças em saúde pública a transmitir convincentemente as relações entre a saúde e outros objetivos sociais, como a prosperidade, competitividade e produtividade. O setor da saúde pública deve representar um papel de facilitador, e deve estar preparado para perceber as prioridades dos setores não ligados a saúde, enxergar como o setor pode dar suporte a estes objetivos e identificar onde há oportunidades estratégicas para a colaboração. Essa recomendação engloba uma metodologia que apresenta diversas estratégias e práticas de governo em diferentes escalas e níveis e serão oportunamente discutidas no decorrer deste trabalho.

Neste estudo analisamos duas situações onde a falta de comunicação, aproximação e engajamento dos diferentes setores e pastas podem comprometer diretamente a saúde da população, além de prejudicar resultados de planos e políticas anteriores. As duas situações selecionadas para este estudo referem-se ao momento da tomada de decisão ou, ao da formulação de uma política pública de saúde específica.

\section{OBJETIVOS}

Os dois objetivos principais deste trabalho são: 1) Apontar a conjuntura de desarticulação entre planejamento, políticas públicas de saúde e meio ambiente com outros setores e pastas estratégicos no Brasil; e, 2) Identificar oportunidades para a aplicação das recomendações da Declaração de Adelaide (WHO, 2010), fomentando a articulação de políticas para o desenvolvimento estratégico do país.

\section{METODOLOGIA}

Este trabalho foi realizado com base na revisão da literatura científica sobre o tema e de documentos governamentais bem como de notícias veiculadas na mídia impressa.

\section{RESULTADOS E DISCUSSÃO}

Embora a saúde seja um dos problemas que mais aflige os brasileiros em todas as regiões do país (Ferreira, 2014) o tema está confinado a sua agenda e há pouquíssima interação e força frente às políticas de outros setores. Diversos são os exemplos, em nosso país, de políticas de saúde que “esqueceram" dos demais determinantes da saúde na sua formulação ou casos em que as questões de 
saúde foram esquecidas na hora da tomada de decisão sobre questões de outros setores cujos impactos afetariam diretamente a saúde de milhares de cidadãos.

\subsection{MEIO AMBIENTE E SAÚDE}

Em 2011, a Organização Mundial da Saúde apontou que as doenças crônicas não transmissíveis serão um dos maiores desafios para o desenvolvimento no século XXI e reconheceu que os governos tem um papel elementar na resposta a este desafio. $O$ aumento na incidência destas doenças afeta principalmente indivíduos de baixa renda e menor escolaridade, pois são estes grupos de indivíduos que estão expostos aos principais fatores de riscos (WHO, 2011). Por incapacitarem os indivíduos estas doenças como mencionado anteriormente apresentam um impacto econômico significativo para o indivíduo e para a sociedade. No Brasil um estudo estimou que a perda de produtividade no trabalho e a diminuição de renda familiar decorrentes da presença de 3 doenças dentro desta classificação (diabetes, AVC e doenças cardiovasculares) podem custar para economia brasileira US\$ 4,18 bilhões no período entre 2006 e 2015 (Abegunde et al., 2007).

No caso das doenças cardiovasculares, câncer de pulmão e doenças neurodegenerativas, diversos estudos apontam o papel determinante de fatores ambientais, tais como a poluição do ar que podem provocar e iniciar o estabelecimento das doenças ou agir como um fator modificador ou de progressão acelerada.

No ano de 2012, um relatório da Organização Mundial de Saúde estimou que no mundo a poluição do ar seja responsável por $16 \%$ das mortes por câncer de pulmão, $11 \%$ das mortes por doenças pulmonares crônicas obstrutivas e mais de $20 \%$ das mortes por doenças isquêmicas do coração e infartos e 13\% das mortes por doenças pulmonares infecciosas (LIM, 2012). A Agência Internacional de Pesquisa sobre o Câncer (IARC) da OMS anunciou em 2013 que há evidencias suficientes de que a poluição do ar causa câncer de pulmão e que há uma associação positiva com um risco aumentado de câncer de bexiga. A avaliação IARC mostrou que há um risco crescente de câncer de pulmão com o aumento dos níveis de exposição a poluição do ar. E os estudos indicam que nos últimos anos os níveis de exposição aumentaram significativamente em algumas partes do mundo, principalmente nos países em processo de industrialização e com grandes populações (IARC, 2013)

Existe uma relação bidirecional entre poluição do ar e fatores de risco cardiovasculares. Indivíduos obesos e diabéticos apresentam maior risco para os efeitos cardiovasculares da exposição e concomitantemente os poluentes do ar podem exacerbar agudamente ou instigar conicamente o desenvolvimento de fatores risco tradicionais. Por exemplo, o componente particulado da poluição do ar provoca elevação na pressão arterial em exposições agudas e as exposições de longo prazo podem 
promover o desenvolvimento de hipertensão arterial crônica. Estes mesmo poluentes também são capazes de prejudicar a sensibilidade a insulina e no longo prazo estas exposições podem promover o desenvolvimento de diabetes mellitus através de respostas inflamatórias sistêmicas (Newby et al, 2015).

A poluição do ar tem amplos efeitos deletérios sobre a saúde humana e é uma questão de saúde importante para a comunidade global. O estudo da carga global de doenças descreveu o impacto da poluição do ar em todo o mundo com responsável por 3,1 milhões de mortes em todas as idades, sendo o total de 52,8 milhões por todas as causas em 2010. Além disso, a poluição atmosférica aparece em nono lugar entre os fatores de risco modificáveis de doença, sendo listado acima de outros fatores comumente reconhecidos, tais como sedentarismo, uma dieta rica em sódio, colesterol alto, e uso de drogas. E a poluição do ar é responsável por 3,1\% dos anos de vida ajustados perdidos por incapacidade, um índice que mede o tempo gasto nos estados de saúde reduzida (Lim et al., 2012)

Assim, baseado nas evidências científicas sobre a importância da qualidade do meio ambiente para a saúde, todas as medidas e planos de ação que visam enfrentar doenças e melhorar a qualidade de vida devem incluir questões relativas a garantia da qualidade do meio ambiente integradamente, tanto por representarem um fator de causa, quanto o de riscos ou situações de agravo para as doenças.

O Brasil elaborou um plano de ação (Plano de Ações Estratégicas para o Enfrentamento das Doenças Crônicas Não-Transmissíveis) para o enfrentamento destas doenças onde aponta que são necessários elos interministeriais que permitam planejamento baseado na avaliação dos impactos de ações fora do setor saúde para que sejam eficazes neste combate. Este plano inclui além das ações diretamente ligadas à saúde (vigilância, atendimento, informação e tratamento) a regulação de setores da sociedade de forma a garantir que interesses de grandes corporações do setor agropecuário e da indústria de alimentos e bebidas estejam voltados também para a prevenção destas doenças (Brasil , 2011; Dunkan et al 2012)

Entretanto, na elaboração deste plano, aspectos determinantes para o estabelecimento e progressão destas doenças ficaram esquecidos. Neste plano de ação a questão da qualidade do meio ambiente, embora apontada como um fator determinante ou modificador de risco para as doenças careceu de ações especificas e restringiu-se apenas às campanhas de sensibilização quanto a promoção de saúde e estímulo a parcerias intersetoriais. Nem mesmo o Ministério do Meio Ambiente é apontado como parceiro desta ação. Isso mostra que planos feitos exclusivamente dentro de alguns setores, sem ou com restrito envolvimento de pesquisadores e profissionais de outras áreas deixa lacunas importantes que poderão comprometer os resultados finais.

O governo brasileiro possui outros louváveis planos de ação de meio ambiente e de saúde, porém carecem de uma intercomunicação mais efetiva. Se considerarmos o exemplo acima e pensarmos sob o ponto de vista da integração da saúde em todas as políticas públicas, poderíamos ter vinculado toda 
essa ação de combate as doenças não transmissíveis aos programas de combate à poluição do ar (PRONAR, PROCONVE, VIGIAR), fortalecendo ambos e diminuindo os gastos e ampliando a abrangência de suas ações. Assim as intervenções para o sucesso de um plano de combate as doenças crônicas não transmissíveis devem ser multifatoriais e amplas o suficiente para que os principais fatores de risco individuais (dieta, sedentarismo, fumo ativo e passivo) e coletivos (poluição do ar) sejam modificados.

\subsection{ECONOMIA, MEIO AMBIENTE E SAÚDE}

Seguindo na temática relacionada à poluição do ar, há um exemplo recente que mostra o desalinhamento entre os setores economia, meio ambiente e saúde. Além disso, reforça a ordem de importância de cada um nas tomadas de decisão, ficando o setor econômico encimado dos demais. Esta questão refere-se ao adiamento da implementação do diesel mais limpo no Brasil.

Nas décadas de 80 e 90 em virtude da expansão da frota de veículos e a crescente emissões de poluentes atmosféricos, sobretudo nas grandes cidades, medidas de controle das emissões veiculares tornaram-se necessárias para que os padrões de qualidade do ar fossem mantidos e a proteção à saúde garantida. Em 06 de maio de 1986, a Resolução nº 18 do CONAMA criou o Programa de Controle de Poluição do Ar por Veículos Automotores - PROCONVE, que definiu os primeiros limites de emissão para veículos, de forma a contribuir para o atendimento aos Padrões de Qualidade do Ar instituídos pelo PRONAR. Assim com o objetivo de diminuir as emissões e promover o desenvolvimento tecnológico nacional de motores e combustíveis uma série de etapas progressivas foram planejadas quanto melhoria que deveria ser alcançada em termos de qualidade dos combustíveis e motores.

A Resolução no 315 de 2002 do CONAMA que regulamentava as novas etapas do Programa de Controle de Emissões Veiculares estabelecia que a partir de 2009, os combustíveis e veículos deveriam ser mais limpos, ou seja, emitir menos poluentes para a atmosfera (equivalentes ao padrão EURO V e diesel com teor de enxofre de 50ppm). Entretanto um ano antes, montadoras e petroquímicas, pressionaram o governo federal a postergar a implementação para 2012 alegando dificuldades tecnológicas e prejuízos econômicos aos estados e municípios, ficando para 2012 a implementação. Apenas as motivações econômicas foram suficientes para que a decisão de prorrogar o novo padrão fosse tomada.

Assim, vale perguntar o seguinte: Quais as implicações desta decisão sobre a saúde da população nas metrópoles, que representam cerca de com quase $37 \%$ da população brasileira (IBGE, 2010), onde se convive com níveis de poluição do ar acima dos limites recomendados pela OMS? E em termos econômicos, qual o custo desse não abatimento nos níveis de poluentes do ar para o sistema de 
saúde público e particular? Se sabemos que as emissões do diesel e de outros combustíveis são prejudiciais à saúde, podendo provocar mortes prematuras e câncer e além disso impactam o meio ambiente, porque estes setores não se posicionaram em defesa do não adiamento da Resolução 315 ?

Em um estudo realizado por nosso grupo de pesquisa na época da tomada de decisão sobre o adiamento, buscamos mostrar que alegações econômicas também poderiam ser utilizadas em favor da saúde de forma a forçar o não adiamento da implementação do novo padrão.

A avaliação do impacto da decisão de adiar a implementação sobre a saúde foi feita estimandose os custos monetários em saúde e à mortalidade associadas a não redução das emissões de MP2.5 no Brasil. O MP2.5 é um dos constituintes da poluição do ar, produzido pela queima incompleta dos combustíveis e a esta fração estão atribuídos os principais efeitos adversos sobre a saúde humana decorrentes da exposição. Por isto este foi o poluente escolhido como referência para aquele estudo.

Durante um ano realizamos campanhas de monitoramento dos níveis de PM2.5 em 6 capitais brasileiras (São Paulo, Rio de Janeiro, Belo Horizonte, Recife, Porto Alegre e Curitiba), cujo objetivo era o de conhecer os níveis ambientais em cada região. Posteriormente, estimamos a redução destes níveis de PM2.5 até o ano de 2040 caso o novo padrão de qualidade do diesel tivesse sido estabelecido, tanto em 2009 quanto em 2012 (ANDRÉ et al. 2012). O impacto sobre a saúde deste não abatimento das emissões de PM2.5 pelo atraso de 2 anos na implementação foi estimado em termos de internações por doenças respiratórias e cardiovasculares separadamente para diferentes faixas etárias, empregando-se coeficientes de estudos séries temporais prévios e também foram expressos em termos de mortalidade como sugerido pela OMS (WHO, 2004).

Por fim, a valoração econômica foi realizada com base nos custos de morbidade, estimados em termos dos custos diretos de internações por problemas respiratórios e doenças cardiovasculares, tanto nos sistema de saúde público quanto privado e, em termos de custos indiretos por perda de produtividade. O custo médio de internações por doenças respiratórias e doenças cardiovasculares para cada faixa etária foi obtido do DATASUS (2009) os custos das internações hospitalares no sistema de saúde privado embora não disponíveis foram considerados três vezes maior com base nos dados do Hospital das Clínicas da Faculdade de Medicina da Universidade de São Paulo. O custo associado a perda da produtividade considera o número de dias de ausência devido a hospitalização (dados obtidos a partir de DATASUS ) e a renda bruta média para cada faixa etária (dados obtidos do IBGE, Censo 2010). Já a valoração econômica de mortalidade foi realizada com base nos anos de vida ajustados por incapacidademétodo DALY (Murray et al., 1997), que combina um fator ambiental com um indicador de saúde para estimar o número de anos de vida perdidos por morte prematura com relação à expectativa de vida no Brasil. 
Os resultados deste estudo revelam que a decisão de adiar a adoção de normas mais restritivas de emissão de poluentes não foi baseada em uma análise séria de custo-benefício ou em uma análise dos impactos sobre a saúde, pois nela não se considerou os custos relacionados à saúde, muito menos os seus benefícios. A não redução das emissões pelo atraso na implementação foi considerada não significativa pelas indústrias e autoridades brasileiras. No entanto, a análise mais detalhada das consequências para a saúde deste atraso, realizado em nosso estudo, indica que devido ao grande número de indivíduos expostos, o atraso na implementação do novo padrão será responsável por um número considerável de internações e um excesso de cerca de 14.000 mortes prematuras até 2040. Os gastos com saúde pública em relação a tal cenário são projetados para aumentar em cerca de US\$ 11,5 bilhões para o mesmo período.

Portanto, questiona-se se as autoridades que aprovaram o adiamento teriam tomado uma decisão diferente se as consequências e os custos para a saúde tivessem sido consideradas no processo de tomada de decisão.

\section{CONSIDERAÇÕES FINAIS}

Há, contudo, um entrave colocado ao debate sobre as políticas públicas, pois são públicas, mas decididas de modo bem particular. A responsabilidade sobre a vida humana deve estar à frente dos interesses econômicos. Isso exige que meio-ambiente como determinante da saúde e a saúde por si sejam levados em consideração nos planos de ação estatal, seja no curto, no médio e no longo prazo. As autoridades governamentais e os grandes empresários devem assumir esse ônus (econômico) em conjunto com a sociedade, redirecionando a política-econômica de suas decisões ao bem da vida e à saúde da população.

A situação de desconsideração das questões de saúde e meio-ambiente podem ser parcialmente explicadas pelo modelo politico-econômico brasileiro que favorece processos produtivos mesmo que estes impliquem em danos direto ou indiretos sobre a saúde e pela falta de preparo técnico de gestores e profissionais da saúde na avaliação dos impactos sobre a saúde das políticas e ações de outros setores e para assumir a necessidade de ações Inter setoriais em todos os planos de ação governamentais.

Nos dois casos perdeu-se a oportunidade de comunicação entre os setores. A interdependência das ações do governo e suas consequências sobre o bem-estar e saúde da população requerem hoje um novo modelo de governança no qual um olhar mais holístico é necessário, o método da Saúde em todas as Políticas seria uma forma, já testada em outros países, para orientar e auxiliar líderes e formuladores de políticas a integrar a o aspecto saúde durante o desenvolvimento, implementação e avaliação das políticas públicas e serviços prestados. 


\section{REFERÊNCIAS}

ABEGUNDE, D.O. et al. The burden and costs of chronic diseases in low-income and middleincome countries. Lancet. v.370, n.9603, p. 1929-38, 207.

ANDRÉ, P.A. et al. Lean diesel technology and human health: a case study in six Brazilian metropolitan regions. Clinics (Sao Paulo). V. 67, n. 6 p. 639-46, 2012.

BRASIL. Ministério da Saúde. Secretaria de Vigilância em Saúde. Departamento de Análise de Situação de Saúde. Plano de ações estratégicas para o enfrentamento das doenças crônicas não transmissíveis (DCNT) no Brasil 2011-2022 / Ministério da Saúde. Secretaria de Vigilância em Saúde. Departamento de Análise de Situação de Saúde. - Brasília : Ministério da Saúde, 2011. 148 p. Disponível em http://actbr.org.br/uploads/conteudo/918_cartilha_dcnt.pdf

DATASUS (Departamento de Informática do SUS). Internações Hospitalares do SUS, 2009. Disponível em: http://tabnet.datasus.gov.br/cgi/deftohtm.exe?sih/cnv/sxsp.def

DUNCAN et al. Doenças crônicas não transmissíveis no Brasil: prioridade para enfrentamento e investigação. Rev. Saúde Pública, São Paulo, v. 46, n.1, p. 126-134 Dec, 2012.

FERREIRA, T. Os problemas que mais afligem os brasileiros - Pesquisa DATFOLHA. Agosto 2014. Disponível em: http://g1.globo.com/jornal-nacional/noticia/2014/08/pesquisa-revela-que-saude-publica-eprincipal-preocupacao-dos-eleitores.html

FRIEL, S. et al. A Public health benefits of strategies to reduce greenhouse-gas emissions: food and agriculture. Lancet. v.374, p. 2016-25, 2009.

HAINES, A. et al. Public health benefits of strategies to reduce greenhouse-gas emissions: overview and implications for policy makers. Lancet. v.374, p. 2104-14, 2009.

IARC. IARC Scientific Publication No. 161 Air Pollution and Cancer. International Agency for Research on Cancer, $2013 . \quad$ Disponível em: http://www.iarc.fr/en/publications/books/sp161/Air\%20Pollution\%20and\%20Cancer.epub

IBGE (Instituto Brasileiro de Geografia e Estatística). Censo 2010 Disponível em: http://censo2010.ibge.gov.br/

LIM, S.S. et al. A comparative risk assessment of burden of disease and injury attributable to 67 risk factors and risk factor clusters in 21 regions, 1990-2010: a systematic analysis for the Global Burden of Disease Study 2010. Lancet. v. 380, n. 9859, p. 2224-60, 2012.

MARKANDYA, A. Public health benefits of strategies to reduce greenhouse-gas emissions: low-carbon electricity generation. Lancet. v.374, p. 2006-15, 2009.

MCGINNIS, J.M. et al. The case for more active policy attention to health promotion. Health Aff (Millwood). v. 21, n.2, p.78-93 Mar-Abr. 2002.

MURRAY, C.J. Regional patterns of disability-free life expectancy and disability-adjusted life expectancy: Global Burden of Disease Study. Lancet. v.349, n. 9062, p. 1347-52, 1997. 
NEWBY, D.E. Expert position paper on air pollution and cardiovascular disease. Eur Heart J. v.36, n. 2, p.83-93 Jan, 2015

STRONG, K. et al. Preventing chronic diseases: how many lives can we save? Lancet. v. 366, p.1578$1582,2005$.

WHO. Second International Conference on Health Promotion. Adelaide Recommendations on Healthy Public Policy. World Health Organization, 1988. Disponível em: http://www.who.int/hpr/archive/docs/adelaide.html

WHO. Adelaide Statement on Health in All Policies Geneva: World Health Organization, 2010. Disponível em: http://www.who.int/social_determinants/hiap_statement_who_sa_final.pdf

WHO. Environmental Burden of Diseases Series N5: Outdoor Air Pollution. World Health Organization. 2004. Disponível em: http://www.who. int/quantifying_ehimpacts/publications/ebd5.pdf

WHO. Macroeconomics and health: investing in health for economic development. Report of the Commission on Macroeconomics and Health. Geneva: World Health Organization; 2001. Disponível em: http://whqlibdoc.who.int/publications/2001/924154550x.pdf

WHO. Health Impact Assessment- The Determinats of Health. World Health Organization, Disponível em: http://www.who.int/hia/evidence/doh/en/ Acesso em Janeiro de 2015.

WHO. Preventing chronic diseases: a vital investment: WHO global report. Geneva, 2005. Disponível em: http://www.who.int/chp/chronic_disease_report/contents/part2.pdf

WHO. United Nations high-level meeting on noncommunicable disease prevention and control: NCD summit to shape the international agenda. World Health Organization. 2011 . Disponível em: http://www.who.int/nmh/events/un_ncd_summit2011/en/

WILKINSON, R.G. et al. Income inequality and population health: a review and explanation of the evidence. Soc Sci Med. v.62, n.7, p.1768-84 Abr. 2006.

WILKINSON, P. et al. Public health benefits of strategies to reduce greenhouse-gas emissions: household energy. Lancet. v.374, p. 1917-29, 2009.

WOODCOCK, J. Public health benefits of strategies to reduce greenhouse-gas emissions: urban land transport. Lancet. v.374, p. 1930-43, 2009. 